ESAIM: PROCEEDINGS, November 2002, Vol.12, 55-60

M.Thiriet, Editor

\title{
CLASSIFICATION OF PERIPHERAL DISTAL BY-PASS GEOMETRIES OBTAINED VIA RECONSTRUCTION FROM MRI.
}

\author{
S. Giordana, J. Peiró, S. Sherwin, D. J. Doorly ${ }^{1}$, Y. Papaharilaou, \\ C. G. CARO ${ }^{2}$ and G. Z. YANG, R. Merrifield ${ }^{3}$
}

\begin{abstract}
This paper discusses the application of a set of computer graphics tools to the reconstruction of three-dimensional models from medical images. Specifically, this work is focused on in-vivo Magnetic Resonance Imaging (MRI) of the distal anastomosis of peripheral by-pass grafts.

The aim of this study is the morphological classification of different anastomoses and the identification of correlations between shape, or more precisely a set of parameters representing shape, and blood flow patterns. Flows patterns are calculated using computational fluid dynamics (CFD) techniques on suitable computer representations of the reconstructed geometries.

The reconstruction of a three-dimensional model of an anastomosis starts from a stack of planar sections of the lumen of the blood vessels produced by segmenting the MR images. An implicit function is fitted through the sections and it is chosen so that its zero-level contour is the interpolating surface of minimum curvature.

A skeletonization algorithm extracts an approximation to the medial line of the threedimensional models. This medial line is used to determine the angles that blood vessels form at the anastomosis. The deviation of the medial line of the grafted vessel from the plane of the anastomosis yields a measure of planarity. These parameters allow a preliminary classification of shape. Scanning the same patient at different times postoperatively also allows to monitor changes in the shape of an anastomosis. Furthermore, scanning the same patient more than once, in different MRI scanners and at different resolutions constitutes an experimental validation of the reconstruction technique.
\end{abstract}

\section{INTRODUCTION}

The occlusion of a peripheral artery in the lower limbs may occur as a consequence of thrombosis or hyperplasia and generally leads to ischemia or gangrene with subsequent limb loss [1]. A peripheral by-pass is a vascular surgery technique where an autologous vein or a prosthetic conduit is grafted to the occluded artery (host artery) in order to allow blood to circumvent the occlusion and re-establish an adequate circulation. The vein is grafted to a healthy artery proximally from the occlusion (proximal anastomosis) and to the occluded artery distally from the occlusion (distal anastomosis). An end-to-side distal anastomosis is the junction of the grafted vein to the host artery that allows blood to flow both proximally and distally from the junction point.

Peripheral by-pass grafts present a high failure rate with the development of myointimal hyperplasia (MIH) in time intervals that can span from as little as a few months to a few years post-operatively [2]. The distal anastomosis seems to be the location that is most afflicted by

\footnotetext{
${ }^{1}$ Department of Aeronautics,Imperial College of Science, Technology and Medicine, London SW7, U.K.

${ }^{2}$ Department of Bioengineering, Imperial College of Science, Technology and Medicine, London SW7, U.K.

${ }^{3}$ Department of Computing, Imperial College of Science, Technology and Medicine, London SW7, U.K.
} 
MIH. There is evidence that the development of MIH within distal end-to-side anastomoses is influenced by the local haemodynamics [3], which is driven by the local geometry and by the characteristics of both the velocity waveform in the graft and the outflow flow split in the host artery. Therefore, the acquisition of in-vivo patient specific data, e.g. MRI and Doppler ultrasound, is a required step when looking into the mechanisms of development of MIH. For example, the capability to monitor the evolution of a single patient's anastomosis with multiple scans allows to look for cause-effect relations between haemodynamic factors computed by CFD and changes in the geometry. On the other hand, accurate CFD simulation of blood flow in a large number of patients requires considerable time and resources, with the further disadvantage of producing large amounts of data that continue to remain patient specific.

More fundamental aspects of blood flow within arterial bifurcations or within by-pass anastomoses can be deduced from CFD simulations and/or experimental measures performed inside idealised tube-like geometries. The construction of meaningful idealised phantoms of a by-pass anastomosis needs to be guided by in-vivo measurements of both flow and geometry. Hence, the acquisition of in-vivo patient specific data and their classification is useful to steer the attention towards more common or more problematic configurations.

The following section presents the reconstruction technique adopted in this work and based on variational radial basis functions. It also shows how the angles used to classify the in-vivo anastomoses do not strongly depend on the smoothness of the reconstructed surface. The Results section discusses some in-vivo applications.

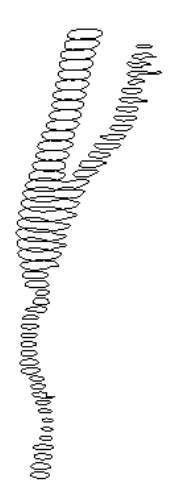

a)

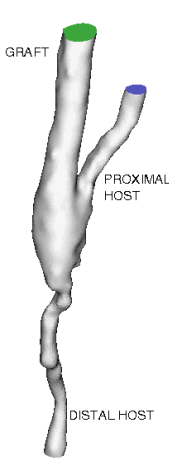

b)

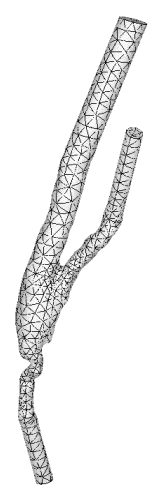

c)

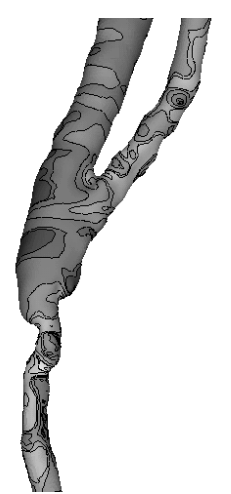

d)

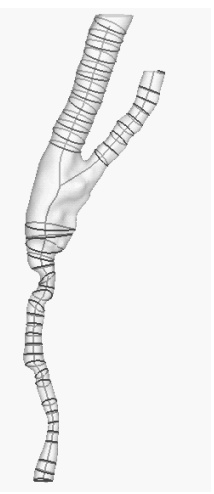

e)

Figure 1. a) Planar contours of the lumen obtained from segmentation of MR images. b) Reconstructed surface. c) High-order discretization. d) Wall shear stress map obtained by high-order CFD. e) Example of medial line and crosssectional area distribution.

\section{Methods}

MR images for each patient involved in this study consist of $512 \times 512$ pixels with their intensity represented on a scale of 256 levels of grey. The MRI scans were obtained from a whole body General Electric 1.5 Tesla scanner with peak gradient strength of $20 \mathrm{mT} / \mathrm{m}$. Images were acquired by MR Angiography pulse sequence based on axial 3D vascular time of flight. The parameters of the acquisitions are: Field of View (FOV) $12 \mathrm{~cm}$, phase FOV 0.8, spatial resolution $256 \times 256$ interpolated to $512 \times 512$ by K-space zero filling, TE $3.5 \mathrm{~ms}$, TR $47 \mathrm{~ms}$, slice thickness $1 \mathrm{~mm}$. The imaging protocol yields areas of blood flow as regions of higher pixel intensity. Images are segmented obtaining the pixelated contours of the lumen of the vessels meeting at the anastomosis. Planar contours are approximated by smooth splines in a least-squares sense, which are then evaluated at discrete points (figure 1a). Typically 30 points per slice are used. These points on the lumenal surface are called surface constraints of the interpolation. More interpolation constraints 
are generated along the in-plane normal to each contour at each of the surface constraints. This new set of points constitutes the normal constraints of the interpolation.

Following Turk and O'Brien [4], the surface reconstruction problem reduces to looking for a function $f$ that assumes a value of 0 on the surface constraints and a unitary value on the normal constraints. An implicit function $f$ that smoothly interpolates the $n$ constraints is defined as

$$
f(\mathbf{x})=\sum_{i=1}^{n} c_{i} \phi\left(\mathbf{x}-\mathbf{x}_{i}\right)
$$

where $\phi(\mathbf{x})$ is a radial basis function and $\mathbf{x}_{i}$ are the interpolation constraints.

Given a family of radial basis functions $\phi(\mathbf{x})$, obtaining the coefficients $c_{i}$ is a matter of solving a linear system $\mathbf{A c}=\mathbf{h}$ that forces $f$ to assume the prescribed values on all the constraints $\mathbf{x}_{i}$, with $a_{i j}=\phi\left(\mathbf{x}_{i}-\mathbf{x}_{j}\right), h_{i}=0$ if $\mathbf{x}_{i}$ is a surface constraint and $h_{i}=1$ if $\mathbf{x}_{i}$ is a normal constraint.

A set of radial basis functions that are suitable for smooth approximations was derived by Duchon [5] in the form of

$$
f(\mathbf{x})=\sum_{i=1}^{n} c_{i}\left\|\mathbf{x}-\mathbf{x}_{i}\right\|^{3}+P(\mathbf{x})
$$

with $P$ a quadratic polynomial. This radial basis minimises a functional that measures curvature in three dimensions. Turk and O'Brien [4] stated that $P$ can be neglected when dealing with more than a dozen of interpolation constraints. The function $f$ as defined by $(2)$ is the multidimensional equivalent of cubic splines that are known to interpolate a set of points while minimising the integral of their second derivative (curvature). The $f=0$ isosurface representing the lumen is extracted by the marching cubes algorithm and consists of a low quality triangulation (figure 1b). This can be re-tiled to obtain a surface mesh suitable for low-order finite volume CFD solvers, or it can be used as the initial support for high-order mesh generation with the purpose of computing a high-order spectral/hp finite element CFD solution (figures $1 \mathrm{c}$ and $1 \mathrm{~d}$ ). The latter allows the exploitation of spectral convergence on unstructured meshes [6]. Should the reconstructed implicit function isosurface require further smoothing, this can be accomplished by applying a non-shrinking Gaussian filter to its triangulation, as described in [7]. A skeletonization algorithm proposed by Palagyi and Kuba [8] is applied to a three-dimensional binary image of the reconstructed surface to extract its medial line (figure 1e).

\subsection{Reconstruction and analysis of a model anastomosis}

A model made up of two tubes joined at an angle of 45 degrees is reconstructed using the implicit function fitting to test the technique. MR images of the models were acquired across 25 planes getting about 16 pixels through the diameter of a slice. Images are segmented using a threshold value of 200. Splines are fitted through each of the 34 cross sections and a set of 40 points are generated on each spline producing a total of 1360 surface constraints. The constraints are interpolated using the implicit function approach and the reconstructed model is shown in figure 2a. The reconstruction shows some artifacts that are present in the MR images, like the undulations that are visible on the lateral surface. A possible explanation of this is a contour shifting due to pixel evaluation. The undulations are easily removed by applying the non-shrinking Gaussian filter (figure $2 \mathrm{~b}$ ). The maximum smoothing that is allowed is such that the distance between the original and the smoothed surface is less than one pixel.

The application of the skeletonization algorithm gives an approximation to the medial line of the tubes. Approximating the medial lines of the three tubes with segments (in a least-squares sense) allows to define the angles at which the three branches meet at the junction (figure 2c). Since the medial lines do not coincide with the axes of the cylinders that generate the model geometry, the angles measured from this linear fit do not intuitively represent the geometry. Better measures are obtained by excluding points on the medial lines that are too close to the junction point (see table 1 and figure $2 \mathrm{~d}$ ). A good criterion to decide which points to disregard is determining a length of reference $L$ as the distance between the junction point and the apex of the junction. This can be done by automatically locating a saddle point on the surface as described in [9]. The exclusion of 
these points leads to measures that are more representative of the angles defined by the axes of the branches of the model bifurcation.

The effect of the smoothing on the measurements can be quantified by measuring the angles on a smoothed reconstruction. Angles computed in the smoothed geometry do not change significantly when compared to those computed in the rough reconstructed surface. This allows us to conclude that smoothing a reconstruction is a necessary operation for a correct representation of a threedimensional model, but it is unnecessary for the determination of the angles from the medial line.

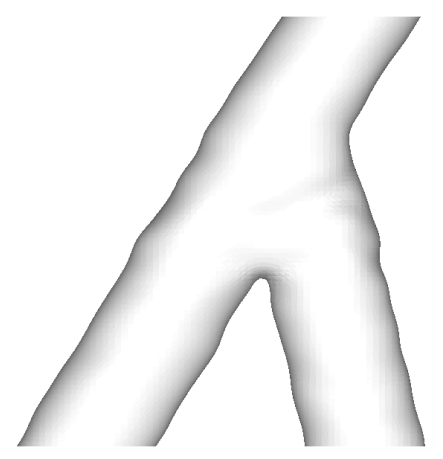

a)

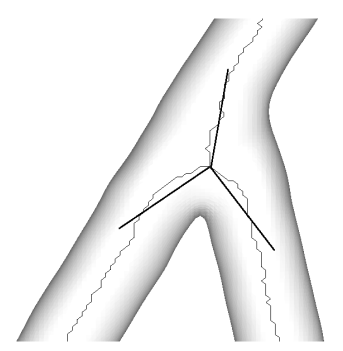

c)

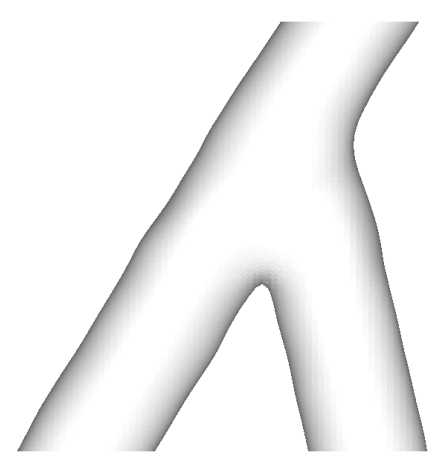

b)

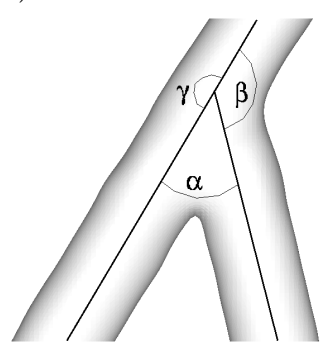

e)

Figure 2. a) Reconstruction of two tubes joined at an angle of 45 degrees. b) Smoothed reconstruction. c) Medial lines of the tubes and their partial approximation by least-squares segments. d) Least-squares approximation of the medial lines outside a region delimited by the length of reference $L$. e) Definition of the three angles reported in table 1.

\begin{tabular}{l|l|l|l} 
Case & $\alpha[\mathrm{deg}]$ & $\beta[\mathrm{deg}]$ & $\gamma[\mathrm{deg}]$ \\
\hline A & 45 & 135 & 180 \\
B & 66 & 134 & 158 \\
C & 45 & 141 & 170 \\
D & 44 & 146 & 167 \\
\hline
\end{tabular}

TABle 1. Angles defined as per figure 2e. Values for Case A are the theoretical angles of the model geometry. Cases B and $\mathrm{C}$ are the un-smoothed reconstruction, all the points on the medial line are used for Case B, while points within $L$ from the junction are disregarded for Case C. Case D refers to the angles measured in the smoothed reconstruction after disregarding points within $L$ from the junction as per figure $2 \mathrm{~d}$. The smoothing has practically no effect on the measurements. 


\section{IN-VIVO APPLICATIONS AND RESULTS}

The first in-vivo example involves the application of the reconstruction technique to multiple scans of the same patient. Figure 3 shows four scans obtained one month, two months, six months and nine months after the operation respectively. The fourth scan was performed in a higher resolution Siemens Sonata scanner. The four reconstructions show that the main features of the vessels are well captured and remain consistent through the various scans. This provides empirical evidence of the reliability of the reconstruction procedure. Monitoring the same patient permits the identification of a progressive stenosis of the proximal host vessel and a reduction of the dimensions of the anastomosis. The ability to detect such features may have significant applications in terms of patient-specific diagnosis and surgical planning. In order to obtain a reduced model of the

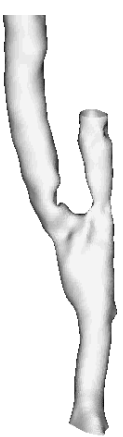

a)

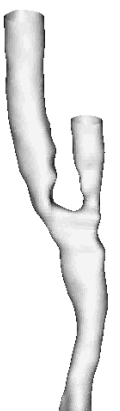

b)

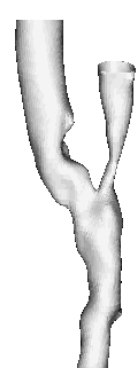

C)

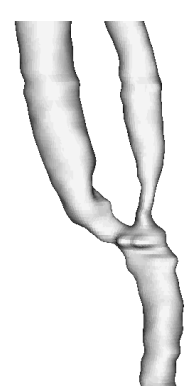

d)

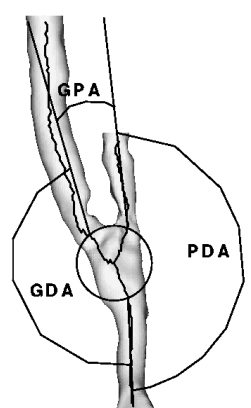

e)

FiguRE 3. a) Reconstruction of an anastomosis imaged one month postoperatively. b) Two months postoperatively. c) Six months postoperatively. d) Nine months postoperatively, confirming the stenosis. e) Definition of the angles used for the classification.

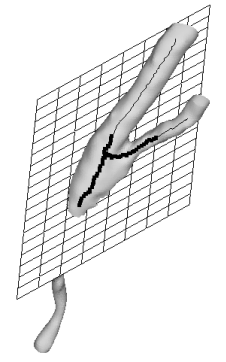

a)

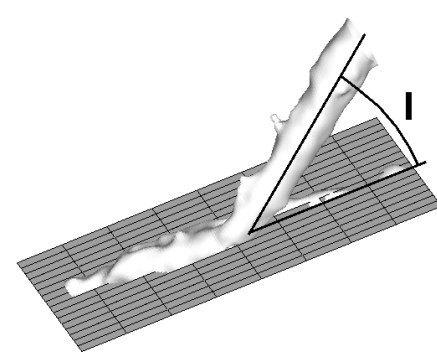

b)

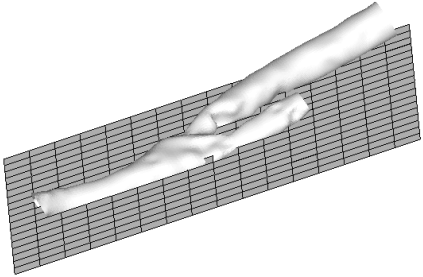

c)

Figure 4. a) Definition of a reference plane. b) Definition of the angle I as a measure of the non-planarity of an anastomosis and example of a non-planar case. I is the angle the graft forms with the reference plane. c) A planar anastomosis.

geometry, three angles can be defined from a linear least squares approximation of the medial line of a reconstructed anastomosis. GPA, GDA and PDA denote the angle between the graft and the proximal host vessel, the graft and the distal host vessel, the proximal and the distal host vessel respectively as shown in figure $3 \mathrm{e}$. The calculated values of the three angles depend on how much information is conveyed into the linear least-squares fit of the medial lines. To compare geometries of different sizes it is necessary to introduce a reference length $\mathrm{D}$ as the mean diameter of the three vessels. This is computed from the cross-sectional area distribution. The three angles are then measured from the directions of three lines interpolating those points on the medial line whose 
distance from the junction is greater than $\mathrm{L}$ and less than 4D. A reference plane is defined as the least-squares plane that fits the points on the medial line belonging to the host vessel within a distance $2 \mathrm{D}$ and those belonging to the graft and within a distance L from the junction (figure 4a). The angle that the graft forms with this plane quantifies the planarity of the anastomosis (figure 4b). The analysis of the angles measured in the anastomoses of different patients allows to identify the most common geometrical configurations. Considering the data obtained from 13 patients scanned up to now, it is possible to identify four families of anastomoses according to the values of GPA and I. The average GPA is 34 degrees although it is mandatory to distinguish between those anastomoses whose GPA is less than 30 degrees and those whose GPA is greater. The same argument applies to I. A wide number of anastomoses are planar (I smaller than the average of 10 degrees) but some show a high level of non-planarity with the greatest I being 32 degrees.

\section{Discussion}

The proposed tools are highly automated and require minimum user intervention. This allows the processing of geometries for a large number of patients in a relatively short time. Furthermore, the higher is the level of automation, the lower is the influence an operator can have on the measurements. The performance of CFD simulation both in anatomically correct and idealised geometries will help identifying which are the key flow features that it is desirable to achieve inside a distal anastomosis. The correlation of these features with simple shape parameters such as the four angles proposed in this paper may allow to help surgeons building more effective anastomoses in the operating theatre.

\section{ACKNOWLEDGEMEnTs}

This work is supported by the Henry Smith's Kensington Estate Charity, The Clothworkers' Foundation and the Garfield Weston Foundation. MR scans are performed at St. Mary's Hospital and at the Royal Brompton Hospital in London. A formal written consent to the treatment of MR images was obtained from all the subjects involved in this study.

\section{REFERENCES}

[1] K. Ouriel. Peripheral arterial disease. The Lancet, 358:1257-1264, 2001.

[2] K. Varty, K. E. Allen, P. R. F. Bell, and N. J. M. London. Infra-inguinal vein graft stenosis. Br. J. Surg., 80:825-833, 1993.

[3] C. G. Caro, J. M. Fitz-Gerald, and R.C. Schroter. Atheroma and arterial wall shear. observations, correlation and proposal of a shear dependent mass transfer mechanism for atherogenesis. Proc. Roy. Soc. B, 177:109-159, 1971.

[4] G. Turk and J. F. O'Brien. Shape transformation using variational implicit surfaces. In Computer Graphics Proceedings, Annual Conference Series, SIGGRAPH99, pages 335-342, Los Angeles, CA, USA, August 8-13 1999. ACM.

[5] J. Duchon. Spline minimizing rotation-invariant semi-norms in Sobolev spaces. In W. Schempp and K. Zeller, editors, Constructive Theory of Functions on Several Variables, number 571 in Lecture Notes in Mathematics, pages 85-99. Springer-Verlag, Berlin, 1977.

[6] G. E. Karniadakis and S. J. Sherwin. Spectral/hp element methods for CFD. Oxford University Press, 1999.

[7] Gabriel Taubin. A signal processing approach to fair surface design. Computer Graphics, 29:351-358, 1995.

[8] K. Palagyi and A. Kuba. A 3d 6-subitration thinning algorithm for extracting medial lines. Pattern Recognition Letters, 19(7):537-641, May 1998.

[9] F. Lazarus and A. Verroust. Level set diagrams of polyhedral objects. Technical Report 3546, INRIA, November 1998. 